\title{
Generalized Bilinear Differential Operators, Binary Bell Polynomials, and Exact Periodic Wave Solution of Boiti-Leon-Manna-Pempinelli Equation
}

\author{
Huanhe Dong, ${ }^{1}$ Yanfeng Zhang, ${ }^{1}$ Yongfeng Zhang, ${ }^{1}$ and Baoshu Yin ${ }^{2,3}$ \\ ${ }^{1}$ College of Mathematics and System Science, Shandong University of Science and Technology, Qingdao 266590, China \\ ${ }^{2}$ Institute of Oceanology, China Academy of Sciences, Qingdao 266071, China \\ ${ }^{3}$ Key Laboratory of Ocean Circulation and Wave, Chinese Academy of Sciences, Qingdao 266071, China \\ Correspondence should be addressed to Yanfeng Zhang; zhangyanfeng0601@163.com
}

Received 5 May 2014; Accepted 20 June 2014; Published 8 July 2014

Academic Editor: Tiecheng Xia

Copyright (C) 2014 Huanhe Dong et al. This is an open access article distributed under the Creative Commons Attribution License, which permits unrestricted use, distribution, and reproduction in any medium, provided the original work is properly cited.

\begin{abstract}
We introduce how to obtain the bilinear form and the exact periodic wave solutions of a class of $(2+1)$-dimensional nonlinear integrable differential equations directly and quickly with the help of the generalized $D_{p}$-operators, binary Bell polynomials, and a general Riemann theta function in terms of the Hirota method. As applications, we solve the periodic wave solution of BLMP equation and it can be reduced to soliton solution via asymptotic analysis when the value of $p$ is 5 .
\end{abstract}

\section{Introduction}

It is significantly important to research nonlinear evolution equations in exploring physical phenomena in depth $[1,2]$. Since the soliton theory has been proposed, the research on seeking the exact solutions of the soliton equations has attracted great attention and made great progress. A series of methods have been proposed, such as Painléve test [3], Bäcklund transformation method [4, 5], Darboux transformation [6], inverse scattering transformation method [7], Lie group method $[8,9]$, and Hamiltonian method $[10,11]$. Particularly, Hirota direct method $[12,13]$ provides a direct approach to solve a kind of specific bilinear differential equations among the exciting methods. As we all know, once the bilinear forms of nonlinear differential equations are obtained, we can construct the multisoliton solutions, the bilinear Bäcklund transformation, and Lax pairs easily. It is clear that the key of Hirota direct method is to find the bilinear forms of the given differential equations by the Hirota differential $D$-operators. Recently, Ma put forward generalized bilinear differential operators named $D_{p}$-operators in
[14] which are used to create bilinear differential equations. Furthermore, different symbols are also used to furnish relations with Bell polynomials in [15], and even for trilinear equations in [16].

In this paper, we would like to explore the relations between multivariate binary Bell polynomials [17-19] and the $D_{p}$-operators and to find the bilinear form of Boiti-LeonManna-Pempinelli (BLMP) equation [20, 21]. Then, we can obtain the exact periodic wave solution [22-25] of the BLMP equation with the help of a general Riemann theta function in terms of Hirota method.

The paper is structured as follows. In Section 2, we will give a brief introduction about the difference between the Hirota differential $D$-operators and the generalized $D_{P^{-}}$ operators. In Section 3, we will explore the relations between multivariate binary Bell polynomials and the $D_{p}$-operators. In Section 4, we will use the relation in Section 2 to seek the differential form of the BLMP equation and then take advantage of the Riemann theta function $[26,27]$ and Hirota method to obtain its exact periodic wave solution which can be reduced to the soliton solution via asymptotic analysis. 


\section{Hirota Bilinear $D$-Operators and the Generalized $D_{p}$-Operators}

It is known to us that Hirota bilinear $D$-operators play a significant role in Hirota direct method. The $D$-operators are defined in [14] as the following:

$$
\begin{aligned}
D_{x}^{m} D_{t}^{n} a(x, t) b(x, t)= & \frac{\partial^{m}}{\partial y^{m}} \frac{\partial^{n}}{\partial y^{n}} \\
& \times\left. a(x+y, t+s) b(x-y, t-s)\right|_{s=0, y=0},
\end{aligned}
$$

where $m, n=0,1,2, \ldots$ Generally, we have

$$
\begin{aligned}
D_{x}^{n} D_{y}^{m} D_{t}^{s} a(x, y, t) b\left(x^{\prime}, y^{\prime}, t^{\prime}\right) \\
=\left(\frac{\partial}{\partial x}-\frac{\partial}{\partial x^{\prime}}\right)^{n}\left(\frac{\partial}{\partial y}-\frac{\partial}{\partial y^{\prime}}\right)^{m} \\
\quad \times\left.\left(\frac{\partial}{\partial t}-\frac{\partial}{\partial t^{\prime}}\right)^{s} a(x, y, t) b\left(x^{\prime}, y^{\prime}, t^{\prime}\right)\right|_{x^{\prime}=x, y^{\prime}=y, t^{\prime}=t},
\end{aligned}
$$

where $m, n, s=0,1,2, \ldots$

For instance, for the Boussinesq equation

$$
u_{t t}-u_{x x}-3\left(u^{2}\right)_{x x}-u_{x x x x}=0
$$

under $u=2(\ln F)_{x x}$, we have

$$
\begin{aligned}
-F_{t}^{2} & +F_{t t} F+F_{x}^{2}+\frac{3}{F^{2}} F_{x}^{4}-F_{x x} F \\
& -\frac{6}{F} F_{x}^{2} F_{x x}+4 F_{x} F_{x x x}-F_{x x x x} F=0 ;
\end{aligned}
$$

we can get its bilinear form with $D$-operators

$$
\left(D_{t}^{2}-D_{x}^{2}-D_{x}^{4}\right) F \cdot F=0 .
$$

However, based on the Hirota $D$-operators, Professor Ma put forward a kind of generalized bilinear $D_{p}$-operators in [14]:

$$
\begin{aligned}
D_{p, x}^{n} D_{p, y}^{m} D_{p, t}^{s} f(x, y, t) g\left(x^{\prime}, y^{\prime}, t^{\prime}\right) \\
=\left(\frac{\partial}{\partial x}+\alpha \frac{\partial}{\partial x^{\prime}}\right)^{n}\left(\frac{\partial}{\partial y}+\alpha \frac{\partial}{\partial y^{\prime}}\right)^{m} \\
\quad \times\left.\left(\frac{\partial}{\partial t}+\alpha \frac{\partial}{\partial t^{\prime}}\right)^{s} f(x, y, t) g\left(x^{\prime}, y^{\prime}, t^{\prime}\right)\right|_{x^{\prime}=x, y^{\prime}=y, t^{\prime}=t},
\end{aligned}
$$

where, for an integer $k$, the $k$ th power of $\alpha$ is defined by

$$
\alpha^{k}=(-1)^{r}(k), \quad \text { if } k \equiv r(k) \bmod p
$$

with $0 \leqslant r(k)<p$.

For example, if $p=2 k(k \in N)$, the $D$-operators are Hirota operators.

If $p=5$, we have

$$
\begin{aligned}
& \alpha=-1, \quad \alpha^{2}=1, \quad \alpha^{3}=-1, \quad \alpha^{4}=\alpha^{5}=1, \\
& \alpha^{6}=-1, \quad \alpha^{7}=1, \quad \alpha^{8}=-1, \quad \alpha^{9}=\alpha^{10}=1,
\end{aligned}
$$

By (6) and (8), it is clear to see that

$$
\begin{gathered}
D_{5, x} D_{5, t} f \cdot g=f_{x t} g-f_{x} g_{t}-f_{t} g_{x}+f g_{x t}, \\
D_{5, x}^{2} f \cdot g=f_{x x} g-2 f_{x} g_{x}+f g_{x x}, \\
D_{5, x}^{4} f \cdot g=f_{4 x}-4 f_{3 x} g_{x}+6 f_{x x} g_{x x}-4 f_{x} g_{3 x}+f g_{4 x} .
\end{gathered}
$$

Now, under $u=2(\ln F)_{x x}$, the generalized bilinear Boussinesq equation can be expressed as

$$
\left(D_{5, t}^{2}-D_{5, x}^{2}-D_{5, x}^{4}\right) F \cdot F=0 .
$$

Then, we would like to discuss how to use the $D$-operator to seek the bilinear differential form of other nonlinear integrable differential equations with the help of binary Bell polynomial.

\section{Binary Bell Polynomial}

As we all know, Bell proposed three kinds of exponentform polynomials. Later, Lambert, Gilson, and their partners generalized the third type of Bell polynomials in [28, 29] which is used mainly in this paper.

The multidimensional binary Bell polynomials which we will use are defined as follows:

$$
\begin{gathered}
Y_{n_{1} x_{1}, \ldots, n_{l} x_{l}}(y)=Y_{n_{1}, \ldots, n_{l}}\left(y_{r_{1}}, \ldots y_{r_{l}}\right) \\
=e^{-y} \partial_{x_{1}}^{n_{1}}, \ldots, \partial_{x_{l}}^{n_{l}} e^{y}\left(n_{1}, \ldots, n_{l} \geq 0\right), \\
\mathscr{Y}_{n_{1} x_{1}, \ldots, n_{l} x_{l}}(v, \omega)=\left.Y_{n_{1}, \ldots, n_{l}}(y)\right|_{\left(y_{r_{1} x_{1}, \ldots, r_{l} x_{l}}=(1 / 2)\left(1+(-1)^{r_{1}+\ldots, r_{l}}\right) v_{r_{1} x_{1}, \ldots, r_{1} x_{1}}+(1 / 2)\left(1-(-1)^{r_{1}+\ldots+r_{l}}\right) \omega_{r_{1} x_{1}, \ldots, r_{1} x_{1}}\right)},
\end{gathered}
$$

in which $y_{r_{1} x_{1}, \ldots, r_{l} x_{l}}=\partial_{x_{1}}^{r_{1}}, \ldots, \partial_{x_{l}}^{r_{l}} y\left(x_{1}, \ldots, x_{l}\right)$. 


$$
\begin{gathered}
y_{x}(v, \omega)=v_{x}, \\
y_{2 x}(v, \omega)=v_{x}^{2}+\omega_{x x}, \\
y_{x, y}(v, \omega)=\omega_{x y}+v_{x} v_{y} .
\end{gathered}
$$

For convenience, we assume that

$$
\begin{aligned}
F=e^{\xi\left(x_{1}, \ldots, x_{l}\right)}, & G=e^{\eta\left(x_{1}, \ldots, x_{l}\right)}, \\
\xi=\frac{\omega+v}{2}, & \eta=\frac{\omega-v}{2}
\end{aligned}
$$

and read that

$$
\begin{aligned}
&(F G)^{-1} D_{p, x_{1}}^{n_{1}}, \ldots, D_{p, x_{l}}^{n_{l}} F \cdot G \\
&= G^{-1}(\eta) F^{-1}(\xi) D_{p, x_{1}}^{n_{1}}, \ldots, D_{p, x_{l}}^{n_{l}} F(\xi) \cdot G(\eta) \\
&= \sum_{k_{1}=0}^{n_{1}} \ldots \sum_{k_{l}=0}^{n_{l}} \prod_{i=1}^{l} \alpha^{k_{i}}\left(\begin{array}{l}
n_{i} \\
k_{i}
\end{array}\right)\left(e^{-\xi} \partial_{x_{1}}^{n_{1}-k_{1}}, \ldots, \partial_{x_{l}}^{n_{l}-k_{l}} e^{\xi}\right) \\
& \times\left(e^{-\eta} \partial_{x_{1}}^{n_{1}-k_{1}}, \ldots, \partial_{x_{l}}^{n_{l}-k_{l}} e^{\eta}\right) \\
&= \sum_{k_{1}=0}^{n_{1}} \ldots \sum_{k_{l}=0}^{n_{l}} \prod_{i=1}^{l}\left(\begin{array}{c}
n_{i} \\
k_{i}
\end{array}\right) Y_{n_{1}-k_{1}, \ldots, n_{l}-k_{l}}(\xi), Y_{k_{1}, \ldots, k_{l}}\left(\alpha^{\sum_{r_{1}}^{r_{l}} r_{i}} \eta\right) \\
&= Y_{n_{1}, \ldots, n_{l}}\left(y_{r_{1}, \ldots, r_{l}}=\xi_{r_{1}, \ldots, r_{l}}+\alpha^{\sum_{r_{1}}^{r_{l}} r_{i}} \eta_{r_{1}, \ldots, r_{l}}\right) \\
&= Y_{n_{1}, \ldots, n_{l}}\left(y_{r_{1}, \ldots, r_{l}}=\frac{1}{2}\left(1+\alpha^{\sum_{r_{1}}^{r_{l}} r_{i}}\right) v_{r_{1}, \ldots, r_{l}}\right. \\
&\left.\quad+\frac{1}{2}\left(1+\alpha^{\sum_{r_{1}}^{r_{l}} r_{i}}\right) \omega_{r_{1}, \ldots, r_{l}}\right) \\
&=\mathscr{Y}_{p ; n_{1} x_{1}, \ldots, n_{l} x_{l}}(v, \omega) .
\end{aligned}
$$

We find that the link between $\mathscr{y}$-polynomials and the $D_{p}$-operator can be given as the following through the above deduction:

$$
\begin{gathered}
\mathscr{Y}_{p ; n_{1} x_{1}, \ldots, n_{l} x_{l}}\left(v=\frac{\ln F}{G}, \omega=\ln F G\right) \\
=Y_{n_{1}, \ldots, n_{l}}\left(y_{r_{1}, \ldots, r_{l}}=\frac{1}{2}\left(1+\alpha^{\sum_{r_{1}}^{r_{l}} r_{i}}\right) v r_{1}, \ldots, r_{l}\right. \\
\left.+\frac{1}{2}\left(1+\alpha^{\sum_{r_{1}}^{r_{l}} r_{i}}\right) \omega r_{1}, \ldots, r_{l}\right) .
\end{gathered}
$$

Particularly, when $F=G$, we define $\mathscr{P}$-polynomials by

$$
\mathscr{P}_{p ; n_{1} x_{1}, \ldots, n_{l} x_{l}}(q)=\mathscr{Y}_{p ; n_{1} x_{1}, \ldots, n_{l} x_{l}}(v=0, \omega=2 \ln F=q) .
$$

When $p=5$, we can obtain that

$$
\begin{aligned}
P_{5 ; y, t} & =q_{x t}, \\
P_{5 ; 2 x} & =q_{x x}, \\
P_{5 ; 4 x} & =3 q_{2 x}^{2}+q_{4 x}, \\
P_{5 ; 3 x, y} & =q_{3 x y}+3 q_{x x} q_{x y}, \\
P_{5 ; 2 x, t} & =0 .
\end{aligned}
$$

Let us now utilize the $\mathscr{P}$-polynomials given above to seek the bilinear form of BLMP equation with the $D_{p}$-operators.

\section{Boiti-Leon-Manna-Pempinelli Equation}

In this section, firstly, we will give the bilinear form of BLMP equation with the help of $\mathscr{P}$-polynomials and the $D_{p^{-}}$ operators. And then, we construct the exact periodic wave solution of BLMP equation with the aid of the Riemann theta function, Hirota direct method, and the special property of the $D_{p}$-operators when acting on exponential functions.

\subsection{Bilinear Form. BLMP equation can be written as}

$$
u_{y, t}+u_{3 x, y}-3 u_{2 x} u_{y}-3 u_{x} u_{x, y}=0 .
$$

Setting $u=-q_{x}$, inserting it into (18), and integrating with respect to $x$ yields

$$
q_{y, t}+q_{3 x, y}+3 q_{x x} q_{x, y}-\lambda=0,
$$

where $\lambda$ is an integral constant.

Based on (17), (19) can be expressed as

$$
P_{5 ; y, t}(q)+P_{5 ; 3 x, y}(q)-\lambda=0 .
$$

From the above, we can get the bilinear form of (18):

$$
\left(D_{5, y} D_{5, t}+D_{5, x}^{3} D_{5, y}\right) F \cdot F-\lambda \cdot F^{2}=0
$$

with $q=2 \ln F$.

4.2. Periodic Wave Solutions. When acting on exponential functions, we find that $D_{p}$-operators have a good property

$$
\begin{aligned}
H & \left(D_{p, x_{1}}, \ldots, D_{p, x_{l}}\right) e^{\xi_{1}} \cdot e^{\xi_{2}} \\
& =H\left(k_{1}+\alpha k_{2}, l_{1}+\alpha l_{2}, w_{1}+\alpha w_{2}\right) e^{\xi_{1}+\xi_{2}},
\end{aligned}
$$

if we assume that

$$
\xi_{i}=k_{i} x+l_{i} y+w_{i} t+\xi_{i}^{(0)} \quad i=1,2, \ldots
$$

As a result of the property above, we consider Riemann's theta function solution of (18):

$$
F=\sum_{n=-\infty}^{\infty} e^{2 \pi i n \eta+\pi i n^{2} \tau},
$$


where $n \in Z, \tau \in C, \operatorname{Im} \tau>0, \eta=k x+l y+w t$, with $k$, $l$, and $w$ being constants to be determined.

Then, we have

$$
\begin{aligned}
& H\left(D_{p, x}, D_{p, y}, D_{p, t}\right) F \cdot F \\
& =\sum_{n=-\infty}^{\infty} \sum_{m=-\infty}^{\infty} H\left(D_{p, x}, D_{p, y}, D_{p, t}\right) e^{2 \pi i n \eta+\pi i n^{2} \tau} e^{2 \pi i m \eta+\pi i m^{2} \tau} \\
& =\sum_{n=-\infty}^{\infty} \sum_{m=-\infty}^{\infty} H(2 \pi i(n+\alpha m) k, 2 \pi i(n+\alpha m) l, \\
& =\sum_{q=-\infty}^{\infty}\left\{\sum _ { m = - \infty } ^ { \infty } H \left(\left(2 \pi i(1-\alpha) n-\alpha^{2} q\right) k,\right.\right. \\
& \quad\left(2 \pi i(1-\alpha) n-\alpha^{2} q\right) l, \\
& \left.\quad\left(2 \pi i(1-\alpha) n-\alpha^{2} q\right) w\right) \\
& \left.\times e^{\pi i\left(n^{2}+(n+\alpha q)^{2}\right) \tau}\right\} e^{2 \pi i(-\alpha q) \eta} \\
& =\sum_{q=-\infty}^{\infty} \bar{H}(q) e^{2 \pi i(-\alpha q) \eta},
\end{aligned}
$$

where $q=-(1 / \alpha)(m+n)$.

To the bilinear form of BLMP equation, $\bar{H}(q)$ satisfies the period characters when $p=5$. The powers of $\alpha$ obey rule (7), noting that

$$
\begin{aligned}
& \bar{H}(q)= \sum_{n=-\infty}^{\infty} H\left(\left(2 \pi i(1-\alpha) n-\alpha^{2} q\right) k\right. \\
&\left(2 \pi i(1-\alpha) n-\alpha^{2} q\right) l, \\
&\left.\left(2 \pi i(1-\alpha) n-\alpha^{2} q\right) w\right) e^{\pi i\left(n^{2}+(n+\alpha q)^{2}\right) \tau} \\
&=\sum_{n=-\infty}^{\infty} H(2 \pi i(2 n-q) k, 2 \pi i(2 n-q) l, \\
&=\sum_{h=-\infty}^{\infty} H(2 \pi i(2 h-(q-2)) k, \\
&2 \pi i(2 h-(q-2)) l, 2 \pi i(2 h-(q-2)) w) \\
& \cdot e^{\pi i\left(h^{2}+(q-h-2)^{2}\right) \tau} \cdot e^{2 \pi i(q-1) \tau} \\
&=\bar{H}(q-2) e^{2 \pi i(q-1) \tau},
\end{aligned}
$$

where $h=n+\alpha$.
From (26) we can infer that

$$
\bar{H}(q)= \begin{cases}\bar{H}(0) e^{\pi i n q \tau}, & q=2 n \\ \bar{H}(1) e^{\pi i\left(2 n+2 n^{2}\right)(q+1) \tau}, & q=2 n+1 .\end{cases}
$$

For (21), we may let

$$
\begin{gathered}
\bar{H}(0)=\sum_{n=-\infty}^{\infty}\left\{[2 \pi i(1-\alpha) n]^{2} l \cdot w+[2 \pi i(1-\alpha) n k]^{3}\right. \\
\cdot 2 \pi i(1-\alpha) n l-\lambda\} e^{2 \pi i n^{2} \tau} \\
=\sum_{n=-\infty}^{\infty}\left(-16 n^{2} \pi^{2} l w+25 n^{4} \pi^{4} k^{3} l-\lambda\right) e^{2 \pi i n^{3} \tau} \\
=0, \quad \cdot \quad 2 \pi\left(i(1-\alpha) n-\alpha^{2}\right) w \\
\bar{H}(1)=\sum_{n=-\infty}^{\infty}\left\{2 \pi\left(i(1-\alpha) n-\alpha^{2}\right) l\right. \\
\quad+\left[2 \pi\left(i(1-\alpha) n-\alpha^{2}\right) k\right]^{3} \\
\left.\quad \cdot 2 \pi\left(i(1-\alpha) n-\alpha^{2}\right) l-\lambda\right\} \\
\quad \times e^{2 \pi i\left(n^{2}-2 n+1\right) \tau} \\
=\sum_{n=-\infty}^{\infty}\left[-4(2 n-1)^{2} \pi^{2} l w+16(2 n-1)^{4} \pi^{4} k^{3} l-\lambda\right] \\
\quad \times e^{\pi i\left(2 n^{2}-2 n+1\right) \tau}=0 .
\end{gathered}
$$

Also, the powers of $\alpha$ obey rule (7). For the sake of computational convenience, we denote that

$$
\begin{aligned}
g_{1}(n) & =e^{2 \pi i n^{2} \tau}, \\
a_{11} & =\sum_{n=-\infty}^{\infty}-16 n^{2} \pi^{2} l g_{1}(n), \\
a_{12} & =\sum_{n=-\infty}^{\infty}\left(256 n^{4} \pi^{4} k^{3} l\right) g_{1}(n), \\
a_{13} & =\sum_{n=-\infty}^{\infty} g_{1}(n) ; \\
g_{2}(n) & =e^{\pi i\left(2 n^{2}-2 n+1\right) \tau}, \\
a_{21} & =\sum_{n=-\infty}^{\infty}-4(2 n-1)^{2} \pi^{2} l g_{2}(n), \\
a_{22} & =\sum_{n=-\infty}^{\infty}\left(16(2 n-1)^{4} \pi^{4} k^{3} l\right) g_{2}(n), \\
a_{23} & =\sum_{n=-\infty}^{\infty} g_{2}(n) .
\end{aligned}
$$


By (28), (29), and (30), we can get that

$$
\begin{aligned}
& a_{11} \omega+a_{12}-\lambda a_{13}=0, \\
& a_{21} \omega+a_{22}-\lambda a_{23}=0 .
\end{aligned}
$$

In view of (30), it is easy to see that

$$
\begin{aligned}
& \omega=\frac{a_{13} a_{22}-a_{23} a_{12}}{a_{11} a_{23}-a_{13} a_{21}}, \\
& \lambda=\frac{a_{12} a_{21}-a_{11} a_{22}}{a_{11} a_{23}-a_{13} a_{21}} .
\end{aligned}
$$

Thus, we obtain the periodic wave solution of BLMP equation:

$$
u=-2(\ln F)_{x}
$$

where $F$ is given by (24) and $\omega, \lambda$ are satisfied with (31).

Then, assuming $e^{\pi i \tau}=\gamma$, based on (29), we may obtain that

$$
\begin{gathered}
a_{11}=\sum_{n=-\infty}^{\infty}-16 n^{2} \pi^{2} l \cdot e^{2 \pi i n^{2} \tau} \\
=-32 \pi^{2} l\left(\gamma^{2}+4 \gamma^{8}+9 \gamma^{18}+\cdots\right), \\
a_{12}=\sum_{n=-\infty}^{\infty} 256 n^{4} \pi^{4} k^{3} l \cdot e^{2 \pi i n^{2} \tau} \\
=2 \times 256 \pi^{4} k^{3} l\left(\gamma^{2}+4 \gamma^{8}+9 \gamma^{18}+\cdots\right), \\
a_{13}=\sum_{n=-\infty}^{\infty} e^{2 \pi i n^{2} \tau}=1+2 \gamma^{2}+2 \gamma^{8}+2 \gamma^{18}+\cdots, \\
a_{21}=\sum_{n=-\infty}^{\infty}-4(2 n-1)^{2} \pi^{2} l \cdot e^{\pi i\left(2 n^{2}-2 n+1\right) \tau} \\
=-8 \pi^{2} l\left(\gamma+9 \gamma^{5}+25 \gamma^{13}+\cdots\right), \\
a_{23}=\sum_{n=-\infty}^{\infty} e^{\pi i\left(2 n^{2}-2 n+1\right) \tau}=1+2 \gamma+2 \gamma^{5}+2 \gamma^{13}+\cdots \\
=\sum_{n=-\infty}^{\infty} 16(2 n-1)^{4} \pi^{4} k^{3} l \cdot e^{\pi i\left(2 n^{2}-2 n+1\right) \tau} \\
=32 \pi^{4} k^{3} l\left(\gamma+3^{4} \gamma^{5}+5^{4} \gamma^{13}+\cdots\right),
\end{gathered}
$$

which lead to

$$
\begin{aligned}
& a_{11} a_{23}-a_{21} a_{13}=8 \pi^{2} l \gamma+o(\gamma), \\
& a_{13} a_{22}-a_{12} a_{23}=32 \pi^{4} k^{3} l \gamma+o(\gamma), \\
& a_{11} a_{22}-a_{12} a_{21}=o(\gamma) .
\end{aligned}
$$

So, we have $\omega \rightarrow 4 \pi^{2} k^{3}$ and $\lambda \rightarrow 0$, as $\gamma \rightarrow 0$.

It is interesting that if we set

$$
\begin{gathered}
k_{1}=2 \pi i k, \quad l_{1}=2 \pi i l, \quad w_{1}=2 \pi i w, \\
\eta_{1}=k_{1} x+l_{1} y+w_{1} t+\pi i \tau,
\end{gathered}
$$

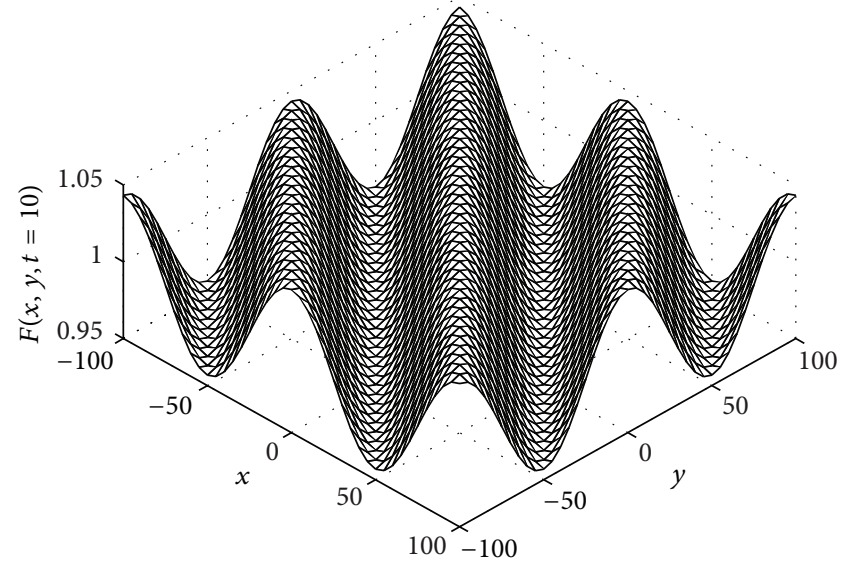

FIgURE 1: Solution of (18).

which can infer that

$$
\begin{aligned}
F & =\sum_{n=-\infty}^{\infty} e^{2 \pi i \eta+\pi i n^{2} \tau}, \\
& =1+e^{\pi i \eta}\left(e^{2 \pi i \eta}+e^{-2 \pi i \eta}\right)+e^{4 \pi i \eta}\left(e^{4 \pi i \eta}+e^{-4 \pi i \eta}\right)+\cdots, \\
& =1+e^{\eta_{1}}+\gamma^{2}\left(e^{\eta_{1}}+e^{2 \eta_{1}}\right)+\gamma^{6}\left(e^{-2 \eta_{1}}+e^{3 \eta_{1}}\right)+\cdots, \\
& \longrightarrow 1+e^{\eta_{1}} \quad\left(\eta_{1} \longrightarrow 0\right) .
\end{aligned}
$$

From all the above, it can be proved that the periodic wave solution (32) just goes to the soliton solution

$$
w_{1}=2 \pi i w \longrightarrow-k_{1}^{3}
$$

Thus, if we assume that $k=0.01, l=0.01$, and $\tau=i$ to the solution

$$
F=1+e^{2 \pi i\left(k x+l y+4 \pi^{2} k^{3} t+\tau\right)}
$$

solution of (18) can be shown in Figure 1.

\section{Conclusions and Remarks}

In this paper, we obtain the bilinear form of bilinear differential equations by applying the $D_{p}$-operators and binary Bell polynomials, which has proved to be a quick and direct method. Furthermore, together with Riemann theta function and Hirota method, we successfully get the exact periodic wave solution and figure of BLMP equation when $p=5$.

There are many other interesting questions on bilinear differential equations, for example, how to apply the generalized operators into the discrete equations; it is known that researches on the discrete and differential equations are also significant. Besides, we will try to explore other operators to construct more nonlinear evolution equations simply and directly in the near future. 


\section{Conflict of Interests}

The authors declare that there is no conflict of interests regarding the publication of this paper.

\section{Acknowledgments}

This work was supported by National Natural Science Foundation of China (no. 11271007), Nature Science Foundation of Shandong Province of China (no. ZR2013AQ017), the Strategic Pioneering Program of Chinese Academy of Sciences (no. XDA 10020104), SDUST Research Fund (no. 2012 KYTD105), Open Fund of the Key Laboratory of Ocean Circulation and Waves, Chinese Academy of Science (no. KLOCAW1401), and Graduate Innovation Foundation from Shandong University of Science and Technology (no. YC130321).

\section{References}

[1] H. W. Yang, B. S. Yin, and Y. L. Shi, "Forced dissipative Boussinesq equation for solitary waves excited by unstable topography," Nonlinear Dynamics, vol. 70, no. 2, pp. 1389-1396, 2012.

[2] H. W. Yang, X. R. Wang, and B. S. Yin, "A kind of new algebraic Rossby solitary waves generated by periodic external source," Nonlinear Dynamics, vol. 76, no. 3, pp. 1725-1735, 2014.

[3] G. Wei, Y. Gao, W. Hu, and C. Zhang, "Painlevé analysis, auto-Bäcklund transformation and new analytic solutions for a generalized variable-coefficient Korteweg-de Vries (KDV) equation," The European Physical Journal B. Condensed Matter and Complex Systems, vol. 53, no. 3, pp. 343-350, 2006.

[4] Y. Yang and Y. Chen, "Pseudopotentials, lax pairs and bäcklund transformations for generalized fifth-order KdV equation," Communications in Theoretical Physics, vol. 55, no. 1, pp. 25-28, 2011.

[5] E. Fan, "New bilinear Bäcklund transformation and Lax pair for the supersymmetric two-BOSon equation," Studies in Applied Mathematics, vol. 127, no. 3, pp. 284-301, 2011.

[6] S. Lou and X. Hu, "Broer-Kaup systems from Darboux transformation related symmetry constraints of KADomtsevPetviashvili equation," Communications in Theoretical Physics, vol. 29, no. 1, pp. 145-148, 1998.

[7] R. Hirota, "The Bäcklund and inverse scattering transform of the K-dV equation with nonuniformities," Journal of the Physical Society of Japan, vol. 46, no. 5, pp. 1681-1682, 1979.

[8] H. Dong and X. Wang, "A Lie algebra containing four parameters for the generalized Dirac hierarchy," Applied Mathematics and Computation, vol. 215, no. 2, pp. 459-463, 2009.

[9] G. W. Bluman and S. Kumei, Symmetries and Differential Equations, Springer, New York, NY, USA, 1989.

[10] Y. Zhang, "A generalized Boite-Pempinelli-Tu (BPT) hierarchy and its bi-Hamiltonian structure," Physics Letters A, vol. 317, no. 3-4, pp. 280-286, 2003.

[11] W. Ma, "Nonlinear continuous integrable Hamiltonian couplings," Applied Mathematics and Computation, vol. 217, no. 17, pp. 7238-7244, 2011.

[12] Y. S. Li, Soliton and Integrable System, Shanghai Scientific and Technological Education Publishing House, Shanghai, China, 1999.
[13] W. Ma, Y. Zhang, Y. Tang, and J. Tu, "Hirota bilinear equations with linear subspaces of solutions," Applied Mathematics and Computation, vol. 218, no. 13, pp. 7174-7183, 2012.

[14] W. Ma, "Bilinear equations, Bell polynomials and linear superposition principle," Journal of Physics: Conference Series, vol. 411, no. 1, Article ID 012021, 2013.

[15] W. X. Ma, "Bilinear equations and resonant solutions characterized by Bell polynomials," Reports on Mathematical Physics, vol. 72, no. 1, pp. 41-56, 2013.

[16] W. Ma, "Trilinear equations, Bell polynomials, and resonant solutions," Frontiers of Mathematics in China, vol. 8, no. 5, pp. 1139-1156, 2013.

[17] W. X. Ma, "Generalized bilinear differential equations," Studies in Nonlinear Sciences, vol. 2, no. 4, pp. 140-144, 2011.

[18] Y. Wang and Y. Chen, "Integrability of an extended (2+1)dimensional shallow water wave equation with Bell polynomials," Chinese Physics B, vol. 22, no. 5, Article ID 050509, 2013.

[19] Y. Wang and Y. Chen, "Binary Bell polynomials, bilinear approach to exact periodic wave solutions of $(2+1)$-dimensional nonlinear evolution equations," Communications in Theoretical Physics, vol. 56, no. 4, pp. 672-678, 2011.

[20] C. R. Gilson, J. J. C. Nimmo, and R. Willox, "A $(2+1)$ dimensional generalization of the AKNS shallow water wave equation," Physics Letters A, vol. 180, no. 4-5, pp. 337-345, 1993.

[21] Q. U. Chang- Zheng, "Symmetry algebras of generalized $(2+$ 1)-dimensional KdV equation," Communications in Theoretical Physics, vol. 25, no. 3, pp. 369-372, 1996.

[22] A. Nakamura, "A direct method of calculating periodic wave solutions to nonlinear evolution equations. I. Exact twoperiodic wave solution," Journal of the Physical Society of Japan, vol. 47, no. 5, pp. 1701-1705, 1979.

[23] T. Xia, B. Li, and H. Zhang, "New explicit and exact solutions for the Nizhnik-NOVikov-Vesselov equation," Applied Mathematics E-Notes, vol. 1, pp. 139-142, 2001.

[24] Z. Zhao, Y. Zhang, and T. Xia, "Double periodic wave solutions of the $(2+1)$-dimensional Sawada-Kotera equation," Abstract and Applied Analysis, vol. 2014, Article ID 534017, 6 pages, 2014.

[25] T. C. Xia, F. J. Yu, and D. Y. Chen, "Multi-component C$\mathrm{KdV}$ hierarchy of soliton equations and its multi-component integrable coupling system," Communications in Theoretical Physics, vol. 42, no. 4, pp. 494-496, 2004.

[26] J. D. Fay, "Riemann's theta function," in Theta Functions on Riemann Surfaces, vol. 352 of Lecture Notes in Mathematics, pp. 1-15, Springer, Berlin, Germany, 1973.

[27] L. Alvarez-Gaumé, G. Moore, and C. Vafa, "Theta functions, modular invariance, and strings," Communications in Mathematical Physics, vol. 106, no. 1, pp. 1-40, 1986.

[28] F. Lambert, I. Loris, J. Springael, and R. Willox, "On a direct bilinearization method: Kaup's higher-order water wave equation as a modified nonlocal Boussinesq equation," Journal of Physics A: Mathematical and General, vol. 27, no. 15, pp. 53255334, 1994.

[29] C. Gilson, F. Lambert, J. Nimmo, and R. Willox, "On the combinatorics of the Hirota D-operators," Proceedings of the Royal Society. London. Series A. Mathematical, Physical and Engineering Sciences, vol. 452, no. 1945, pp. 223-234, 1996. 


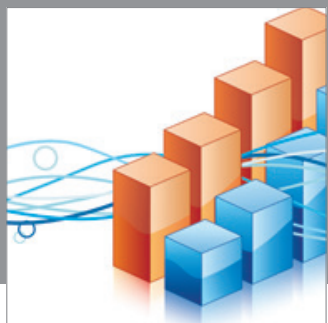

Advances in

Operations Research

mansans

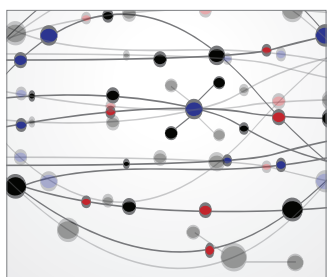

The Scientific World Journal
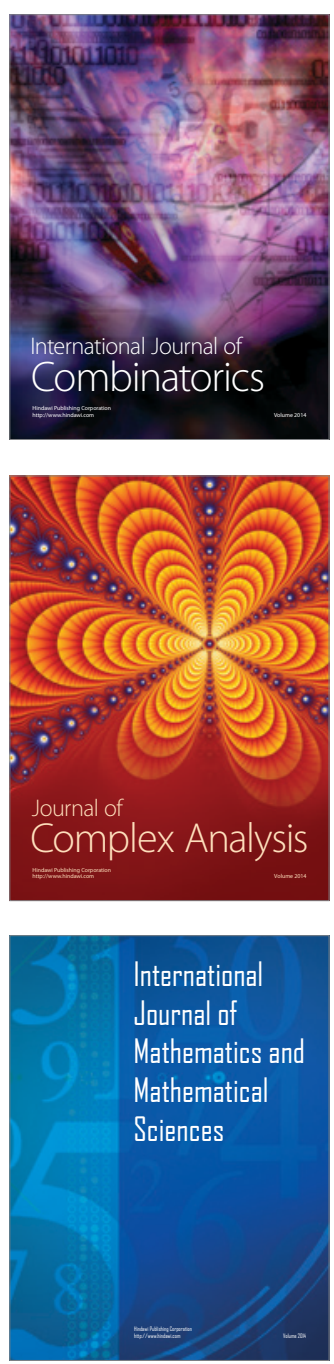
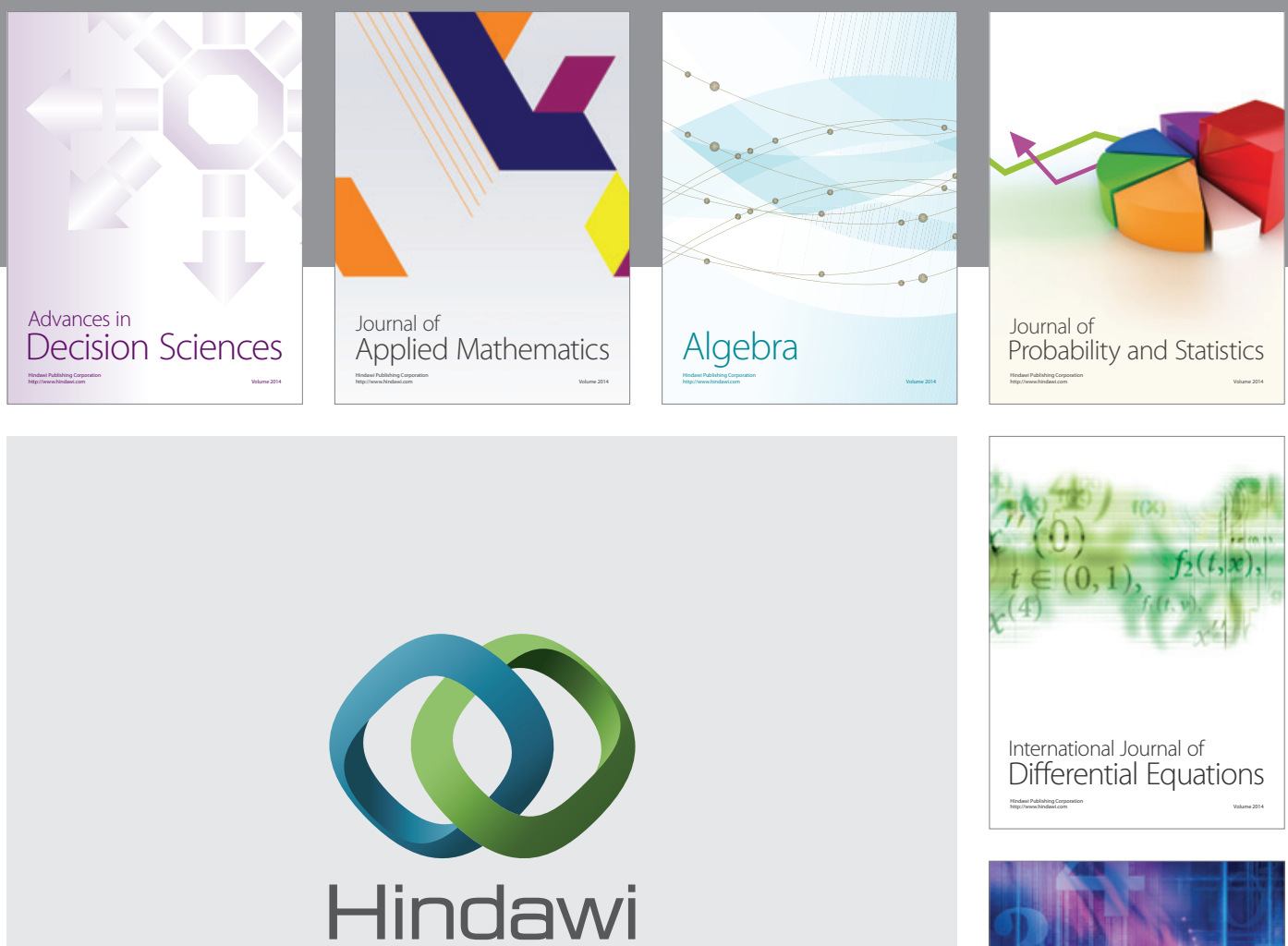

Submit your manuscripts at http://www.hindawi.com
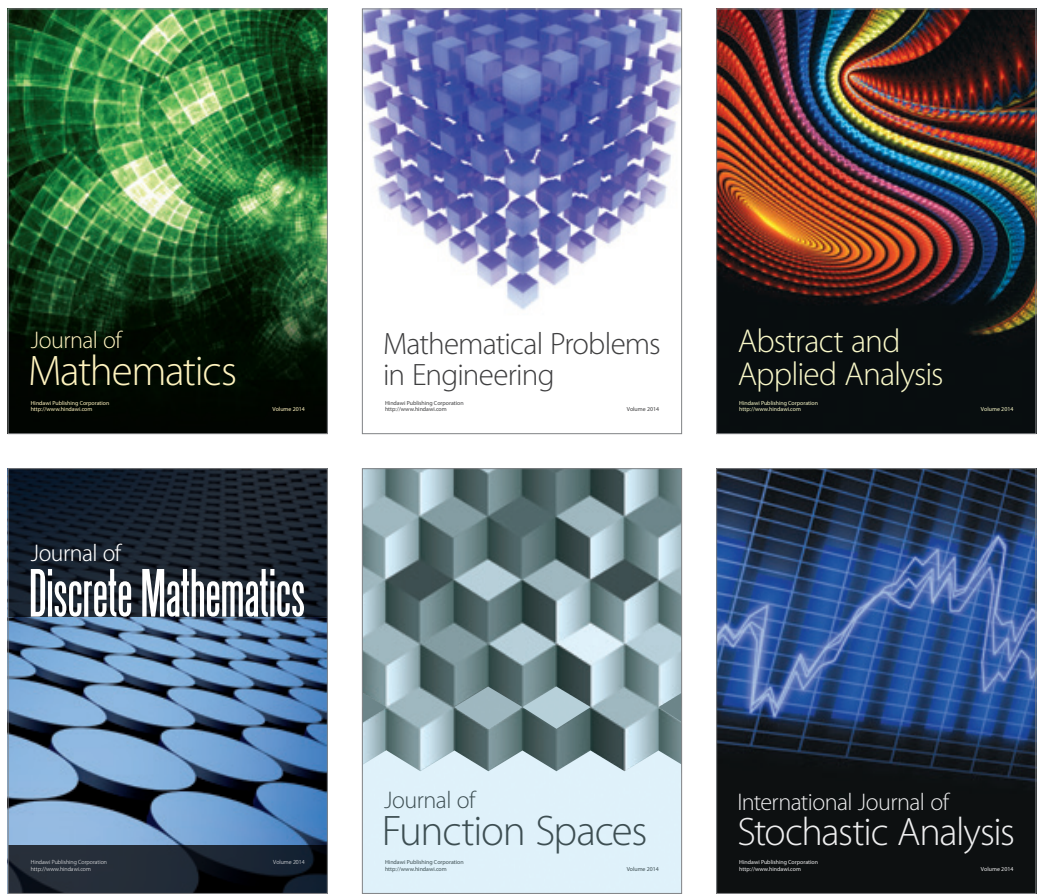

Journal of

Function Spaces

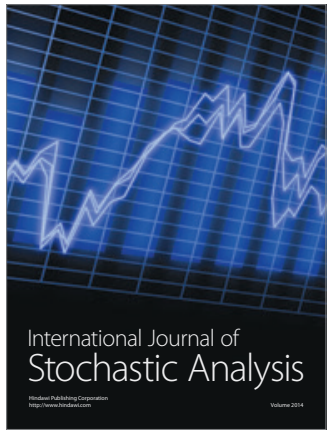

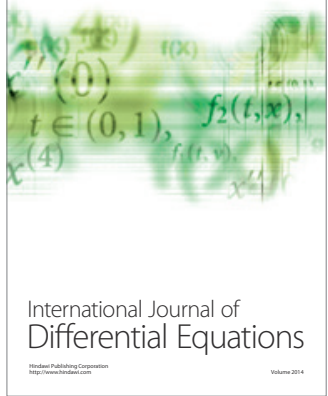
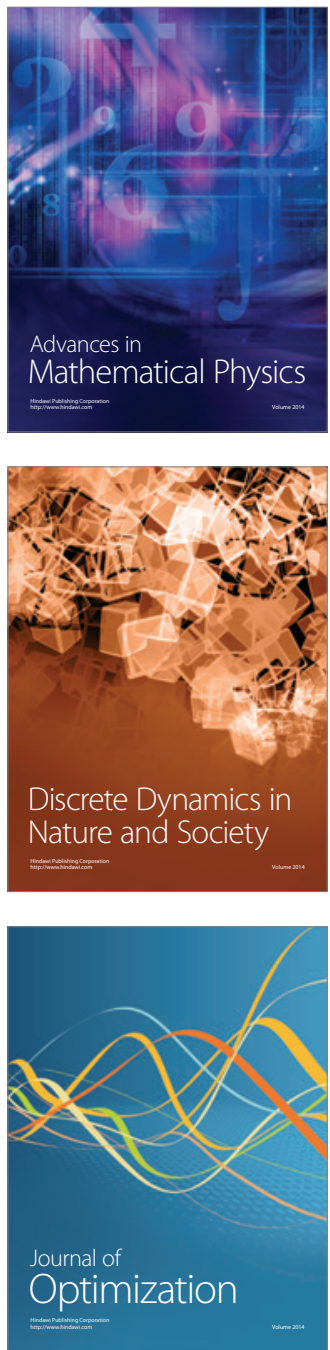